\title{
Oxidative Dimer Produced from a 2,3,4-Trihydroxybenzoic Ester
}

\author{
Asuka Kodama, Hidetoshi Shibano, and Jun Kawabata ${ }^{\dagger}$ \\ Laboratory of Food Biochemistry, Division of Applied Bioscience, Graduate School of Agriculture, \\ Hokkaido University, Sapporo 060-8589, Japan
}

Received March 12, 2007; Accepted April 2, 2007; Online Publication, July 7, 2007

[doi:10.1271/bbb.70139]

The DPPH radical-scavenging abilities of the naturally occurring phenolic acid, 2,3,4-trihydroxybenzoic acid, and its methyl ester were evaluated. Both compounds in acetonitrile scavenged as many as four radicals compared to three or fewer radical consumption in acetone or ethanol. Only the ester showed relatively high ability in methanol. Oxidation with $o$-chloranil in acetonitrile resulted in methyl 2,3,4-trihydroxybenzoate giving a novel benzocoumarin-type dimer, its chemical structure being confirmed by spectroscopic evidence. The formation of this dimer might partly account for the higher radical-scavenging efficiency of the ester in acetonitrile or methanol.

Key words: 2,3,4-trihydroxybenzoic acid; phenolic acid; oxidative coupling; DPPH radical; $o$-chloranil

Hydroxybenzoic acids and esters are widely distributed in plants and have attracted considerable attention for their antioxidative activities. ${ }^{1-4)}$ The radical-scavenging reaction of catechol- and pyrogallol-type hydroxybenzoic acids typically comprises two distinctive phases, initially fast and subsequently slow., ${ }^{5,6)}$ The former phase is recognized as the conversion of an $o$-diphenol to the corresponding $o$-quinone, whereas complex reactions including oligomerization of the resulting quinone would occur in the latter slow phase. The difference in total radical-scavenging efficiency of each hydroxybenzoic acid inherently depends on this latter phase, although little has been found for this complex stage. So far, a protocatechuic (3,4-dihydroxybenzoic) ester (1) gave its quinone dimer, ${ }^{6}$ quinone acetal $^{7)}$ and adducts, ${ }^{8-11)}$ and a gallic (3,4,5-trihydroxybenzoic) ester (2) and its analogs also produced quinone dimers ${ }^{6,12,13)}$ and adducts. ${ }^{12)}$ In contrast to protocatechuic and gallic acids, there has been no report on the oxidation reaction of another naturally occurring phenolic acid, 2,3,4trihydroxybenzoic acid (3). ${ }^{14-16)}$ We have investigated the DPPH (2,2-diphenyl-1-picrylhydrazyl) radicalscavenging abilities and the oxidant-mediated reaction of 2,3,4-trihydroxybenzoic acid (3) and its ester (4)

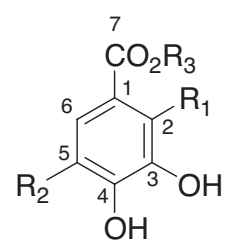

$$
\begin{aligned}
& \text { 1: } \mathrm{R}_{1}=\mathrm{R}_{2}=\mathrm{H}, \mathrm{R}_{3}=\mathrm{CH}_{3} \\
& \text { 2: } \mathrm{R}_{1}=\mathrm{H}, \mathrm{R}_{2}=\mathrm{OH}, \mathrm{R}_{3}=\mathrm{CH}_{3} \\
& \text { 3: } \mathrm{R}_{1}=\mathrm{OH}, \mathrm{R}_{2}=\mathrm{R}_{3}=\mathrm{H} \\
& \text { 4: } \mathrm{R}_{1}=\mathrm{OH}, \mathrm{R}_{2}=\mathrm{H}, \mathrm{R}_{3}=\mathrm{CH}_{3}
\end{aligned}
$$

Fig. 1. Structures of 2,3,4-Trihydroxybenzoic Acid and Related Compounds.

(Fig. 1), and identified a novel oxidative dimer in the reaction mixture with $o$-chloranil (3,4,5,6-tetrachloro- $o$ benzoquinone) in acetonitrile.

\section{Materials and Methods}

Reagents. 2,3,4-Trihydroxybenzoic acid (3) (Tokyo Kasei Kogyo Co.), 2,2-diphenyl-1-picrylhydrazyl (DP$\mathrm{PH}$ ) radical (Wako Pure Chem. Ind.) and $o$-chloranil (Aldrich Chem. Co.) were purchased from each supplier. Methyl 2,3,4-trihydroxybenzoate (4) was prepared from 3 by heating with methanol containing $10 \%$ hydrogen chloride. All solvents used were of technical grade.

Colorimetric radical-scavenging tests. DPPH radical scavenging tests were conducted as described in the previous paper. ${ }^{6}$ )

HPLC analysis. A mixture of phenols $(0.025 \mathrm{mmol})$ and the DPPH radical $(0.05$ or $0.1 \mathrm{mmol})$ or $o$-chloranil $(0.025$ or $0.05 \mathrm{mmol})$ in acetonitrile $(0.4 \mathrm{ml})$ was analyzed 5 and $70 \mathrm{~min}$ after mixing. The analytical conditions were as follow: column, Inertsil PREP-ODS $(6.0 \times$ $250 \mathrm{~mm}$, GL Sciences); mobile phase, $4 \%$ (0-5 min), 4$16 \%$ (5-20 min), 16-32\% (20-35 min), 32-80\% (35$45 \mathrm{~min})$ and $80 \%$ (45-60 min) acetonitrile in water containing $2.5 \%$ acetic acid; flow rate, $1.0 \mathrm{ml} / \mathrm{min}$; detec-

\footnotetext{
$\dagger$ To whom correspondence should be addressed. Fax: +81-11-706-2496; E-mail: junk@chem.agr.hokudai.ac.jp
} 
tion, UV 260 or $400 \mathrm{~nm}$. Under these conditions, 3 and 4 were eluted at $t_{\mathrm{R}}=23.4$ and $37.5 \mathrm{~min}$, respectively.

Isolation of benzocoumarin dimer 5 from the reaction of methyl 2,3,4-trihydroxybenzoate (4) with o-chloranil. To a solution of $4(23 \mathrm{mg}, 0.13 \mathrm{mmol})$ in acetonitrile $(1 \mathrm{ml})$ was added $o$-chloranil $(31 \mathrm{mg}, 0.13 \mathrm{mmol})$ in acetonitrile $(1 \mathrm{ml})$. After $16 \mathrm{hr}$ at room temperature, a dark brown precipitate appearing in the reaction mixture was separated and washed with acetonitrile to give a pure dimer (5) as dark brown powder (4 mg, 18\%). 5. $\mathrm{Mp}>300^{\circ} \mathrm{C}$; FD-MS m/z (\%): 334 (100); EI-HR-MS $m / z: 334.0334\left([\mathrm{M}]^{+}\right.$, calcd for $\left.\mathrm{C}_{15} \mathrm{H}_{10} \mathrm{O}_{9}, 334.0325\right)$; ${ }^{1} \mathrm{H}-\mathrm{NMR} \delta$ (DMSO- $\left.d_{6}\right)$ ppm: $3.94\left(3 \mathrm{H}, \mathrm{s}, \mathrm{H}-\mathrm{OCH}_{3}\right)$, 7.09 (1H, s, H-5') 7.86 (1H, s, H-6), $9.17\left(1 \mathrm{H}, \mathrm{s}, \mathrm{OH}-3^{\prime}\right)$, $9.86(1 \mathrm{H}, \mathrm{s}, \mathrm{OH}-3), 10.57(1 \mathrm{H}, \mathrm{s}, \mathrm{OH}-2), 10.58(1 \mathrm{H}, \mathrm{s}$, OH-4' $10.98\left(1 \mathrm{H}, \mathrm{s}, \mathrm{OH}-2^{\prime}\right) ;{ }^{13} \mathrm{C}-\mathrm{NMR} \delta\left(\mathrm{DMSO}-d_{6}\right)$ ppm: $52.7\left(\mathrm{C}-\mathrm{OCH}_{3}\right), 98.0\left(\mathrm{C}-1^{\prime}\right), 99.8\left(\mathrm{C}-5^{\prime}\right), 110.7(\mathrm{C}-$ 1), 110.8 (C-5), 113.3 (C-6), 126.5 (C-6'), 132.7 (C-3'), 133.6 (C-3), 142.5 (C-4), 149.4 (C-2), 150.0 (C-2'), $154.6\left(\mathrm{C}-4^{\prime}\right), 164.0\left(\mathrm{C}-7^{\prime}\right), 168.7$ (C-7); HMBC correlation: $\mathrm{H}-\mathrm{OCH}_{3} / \mathrm{C}-7, \mathrm{H}-5^{\prime} / \mathrm{C}-5,1^{\prime}, 3^{\prime}, 4^{\prime}$ and $7^{\prime}, \mathrm{H}-6 / \mathrm{C}-$ 2, 4, 7 and $6^{\prime}, \mathrm{OH}-3^{\prime} / \mathrm{C}-2^{\prime}, \mathrm{OH}-3 / \mathrm{C}-2$ and $4, \mathrm{OH}-2 / \mathrm{C}-1$, 2 and $3, \mathrm{OH}-4^{\prime} / \mathrm{C}-3^{\prime}$ and $5^{\prime}, \mathrm{OH}-2^{\prime} / \mathrm{C}-1^{\prime}, 2^{\prime}$ and $3^{\prime}$.

\section{Results and Discussion}

The DPPH radical-scavenging abilities of 2,3,4trihydroxybenzoic acid (3) and methyl 2,3,4-trihydroxybenzoate (4) were determined by the colorimetric method. After $30 \mathrm{~min}$, the relative radical-scavenging equivalence of each compound, when that of $\alpha$-tocopherol in ethanol as standard was designated as $2,{ }^{17,18)}$ was as follows: $\mathbf{3}, 4.3$ and $\mathbf{4}, 3.7$ in acetonitrile; $\mathbf{3}, 3.1$ and 4, 2.1 in acetone; 3, 2.9 and 4, 4.7 in methanol; and 3, 2.7 and 4, 3.2 in ethanol. The radical-scavenging ability of $\mathbf{3}$ was highest in acetonitrile and decreased in the order of acetonitrile $>$ acetone $>$ methanol $>$ ethanol, whereas methyl ester 4 showed the order of methanol $>$ acetonitrile $>$ ethanol $>$ acetone. Unlike the protocatechuic esters, the DPPH radical-scavenging equivalence of 2,3,4-trihydroxybenzoic acid (3) and its ester (4) did not show any marked solvent dependence, without any difference in alcoholic and non-alcoholic solvents. ${ }^{9)}$ The radical-scavenging equivalence of $\mathbf{3}$ and 4 in acetonitrile exceeded three, whereas nearly three or fewer radicals were consumed in other solvents, except with 4 in methanol. These results indicate that a further oxidative reaction might have occurred in acetonitrile, since only three radicals were expected to be scavenged during the simple quinone-oxy radical formation from pyrogallols.

An HPLC analysis of the reaction mixture of $\mathbf{3}$ or $\mathbf{4}$ with the DPPH radical in acetonitrile, however, gave no significant peak of an oxidation product. Oxidation mechanism studies on polyphenols have frequently used $o$-chloranil as an oxidant from a catechol to the corresponding quinone, since the reaction proceeds cleanly compared to using radical species. ${ }^{12,13,19)}$ Changing the oxidant from the DPPH radical to $o$-chloranil could simplify the chromatogram of the reaction mixture, probably due to the mild oxidative property of the nonradical chlorinated quinone compared to the DPPH radical. Hence, a distinctive new peak $\left(t_{\mathrm{R}}=43.5 \mathrm{~min}\right)$ was observed in the equimolar reaction mixture of $\mathbf{4}$ and $o$-chloranil in acetonitrile at $5 \mathrm{~min}$, as well as a broad peak $\left(t_{\mathrm{R}}=23.6 \mathrm{~min}\right)$ detected at $400 \mathrm{~nm}$. The former peak increased in size during $70 \mathrm{~min}$ after the reaction had started. These two peaks were also observed in the corresponding reaction mixture in methanol. In addition, a dark brown precipitate appeared in the acetonitrile reaction solution after several hours. The precipitated product was filtered off and directly subjected to instrumental analyses, which unveiled that it was a novel benzocoumarin-type coupling product (5) derived from two molecules of 4. The mass spectral analysis gave a molecular peak at $m / z$ 334, and the high-resolution analysis indicated the molecular formula of $\mathrm{C}_{15} \mathrm{H}_{10} \mathrm{O}_{9}$, which corresponded to a loss of $\mathrm{CH}_{3} \mathrm{OH}$ and $2 \mathrm{H}$ from two molecules of $\mathbf{4}$. The ${ }^{1} \mathrm{H}-\mathrm{NMR}$ spectrum of $\mathbf{5}$ gave a relatively simple peak pattern. Only two singlet signals of aromatic protons at $\delta 7.09$ and 7.86, as well as a methoxyl signal at $\delta 3.94$ were observed, apart from five exchangeable hydroxyl protons in the low-field region of $\delta 9-11$. In the ${ }^{13} \mathrm{C}-\mathrm{NMR}$ spectrum, 15 carbon signals, including two ester carbonyls at $\delta 164.0$ and 168.7 and one methoxyl at $\delta 52.7$, were observed. In the HMBC spectrum, the lower-field aromatic proton of $\delta 7.86$ showed correlation peaks with two oxycarbons at $\delta 142.5$ and 149.4 , as well as one of the ester carbonyls at 168.7 (C-7), and was thus assigned to H-6 of one unit of 4. On the other hand, the other aromatic proton at $\delta 7.09$ had a correlation peak with one upfield-shifted oxycarbon at $\delta$ 132.7, being assignable to the central oxygenated carbon $\left(\mathrm{C}-3^{\prime}\right)$ of a pyrogallol structure. This proton and the other ester carbonyl of $\delta 164.0$ (C-7') gave a weak cross peak, suggesting their $\mathrm{W}$-shaped long-range relationship. Hence, the higher-field proton could be reasonably assigned as $\mathrm{H}_{-} 5^{\prime}$ of the second unit of 4 . The signal of H-6 showed an additional strong three-bond cross peak with a quaternary carbon at $\delta 126.5$, and similarly, $\mathrm{H}-5^{\prime}$ also had a cross peak with another quaternary carbon at $\delta$ 110.8. The former quaternary carbon was reasonably assigned as $\mathrm{C}-6^{\prime}(\Delta \delta+13.2)$, and the latter as $\mathrm{C}-5(\Delta \delta$ $+11.0)$, since the chemical shifts of those carbons were equally shifted downfield compared to unsubstituted C-6 ( $\delta$ 113.3) and $C-5^{\prime}$ ( $\delta$ 99.8), respectively, by their aryl substitution. These HMBC correlations well support the connectivity between $\mathrm{C}-5$ of the first unit and C- $6^{\prime}$ of the second unit. The connection of C-5 and C- $6^{\prime}$ enabled $\delta$ lactone formation between $\mathrm{OH}-4$ of the former unit and the carboxyl of the latter. A detailed analysis of the HMBC correlations unambiguously confirmed the total structure of 5 (Fig. 2).

Benzocoumarin 5 comprises two molecules of $\mathbf{4}$ by connecting between C-5 of one unit and C- 6 of the other. 


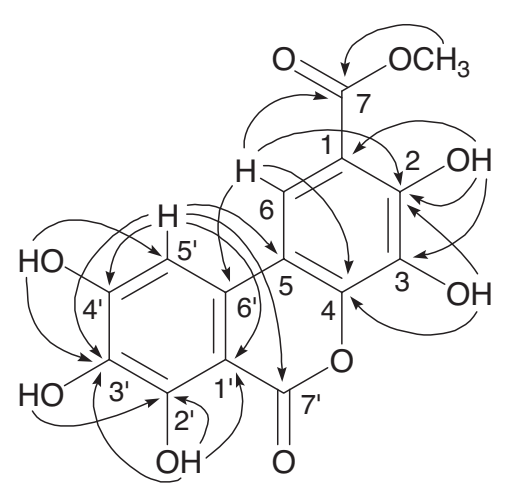

Fig. 2. HMBC Correlations in $\mathbf{5}$.

First, 4 is oxidized to give the corresponding 3,4quinone (4q), since $\mathrm{OH}-2$ could hardly be oxidized by intramolecular hydrogen bonding with an ester carbonyl. Then, nucleophilic attack on C-6 of $\mathbf{4 q}$ with C-5 of $\mathbf{4}$, which is a doubly activated position by para-OH-2 and ortho-OH-4, occurs and subsequent deprotonation and lactonization would afford 5 (Fig. 3). The sharp peak eluted at $t_{\mathrm{R}}=43.5 \mathrm{~min}$ in the HPLC analysis could be assigned as benzocoumarin $\mathbf{5}$, and the broad peak at $t_{\mathrm{R}}=23.6 \mathrm{~min}$ detected at the longer wavelength of $400 \mathrm{~nm}$ was probably due to quinone 4q. When increasing the molar ratio of $o$-chloranil against $\mathbf{4}$ to 2:1, the peak of 5 diminished after $70 \mathrm{~min}$ of reaction, although it was visible at $5 \mathrm{~min}$, which suggests that further oxidation of $\mathbf{5}$ to the corresponding quinone or equivalents might occur with excess oxidant. In the case of 3, similar oxidative coupling could occur in the reaction with $o$-chloranil, although the detection of any corresponding HPLC peak failed and no such precipitate as that found in the reaction of $\mathbf{4}$ appeared in the reaction mixture.

In alcoholic solvents, the nucleophilic addition of an alcohol molecule to the resulting quinone would predominate, although no methanol adduct was actually identified from the reaction mixture of $\mathbf{4}$ with the DPPH radical or $o$-chloranil in methanol due to the complexity of the oxidation reaction of pyrogallol-type ester $\mathbf{4}$ compared to catechol-type ester 1 . The rate of the addition could directly reflect the radical-scavenging equivalence of $\mathbf{3}$ and $\mathbf{4}$ in methanol or ethanol. The highest equivalence (4.7) obtained with the combination of $\mathbf{4}$ and methanol well corresponds to the result from the high reactivity in methyl protocatechuate (1) and methanol. ${ }^{9)}$ The relatively low reactivity of $\mathbf{3}$ (2.9) compared to $\mathbf{4}$ can also be accounted for by conversion to the less reactive carboxylate form of the quinone, as has been seen in the case of protocatechuic acid. ${ }^{20)}$

On the other hand, in non-alcoholic solvents, any direct participation of the solvent molecule in the reaction must be negligible. Hence, the high radicalscavenging equivalence of $\mathbf{3}$ (4.3) and 4 (3.7) in acetonitrile could be due to the ability to produce a coupling product such as $\mathbf{5}$. This type of dimeric product might easily undergo a further oxidation reaction by consuming more radicals to give complex products. The higher dielectric constant of acetonitrile (36.6) compared to less polar acetone (20.7) could be favorable for the effective molecular stacking of hydrophobic $\mathbf{4}$ and 4q. Unlike the reactivity in alcoholic solvents, free acid 3 showed higher radical-scavenging activity than its ester 4 in non-alcoholic solvents. Although the reason is unknown at this stage, the coupling reaction of $\mathbf{3}$ and its quinone may be rapid enough to give polymeric products, this being supported by the failure to identify the acid derivative of $\mathbf{5}$ from the reaction mixture of $\mathbf{3}$ in methanol.

In conclusion, benzocoumarin dimer $\mathbf{5}$ produced in the reaction mixture of methyl 2,3,4-trihydroxybenzoate 4 with $o$-chloranil in acetonitrile or methanol is a novel type of oxidative coupling product of phenolic acids, and a similar coupling reaction by other polyphenols may proceed in suitable reaction media.

\section{Acknowledgments}

We are grateful to Mr. Kenji Watanabe and Dr. Eri Fukushi of our school for their skilful measurement of the mass spectra. This work was supported partly by the Japan Food Chemical Research Foundation.

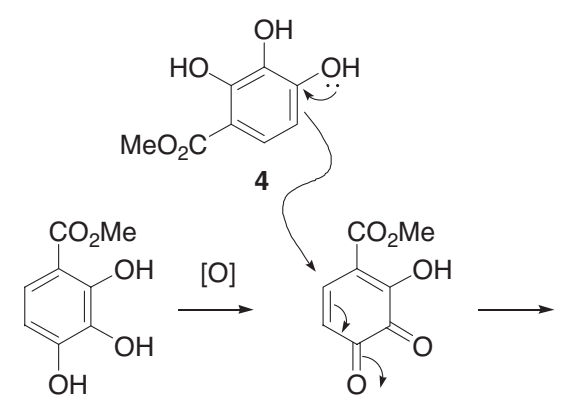

4

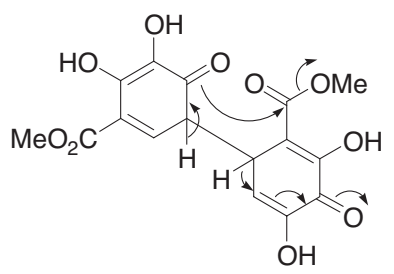

$4 q$<smiles>CC(C)=O</smiles>

5

Fig. 3. Plausible Oxidation Pathway of 4. 


\section{References}

1) Cuvelier, M.-E., Richard, H., and Berset, C., Comparison of the antioxidative activity of some acid-phenols: structure-activity relationship. Biosci. Biotechnol. Biochem., 56, 324-325 (1992).

2) Rice-Evans, C. A., Miller, N. J., and Paganga, G., Structure-antioxidant activity relationships of flavonoids and phenolic acids. Free Radic. Biol. Med., 20, 933-956 (1996).

3) Natella, F., Nardini, M., Di Felice, M., and Saccini, C., Benzoic and cinnamic acid derivatives as antioxidants: structure-activity relation. J. Agric. Food Chem., 47, 1453-1459 (1999).

4) Tyrakowska, B., Soffers, A. E. M. F., Szymusiak, H., Boeren, S., Boersma, M. G., Lemanska, K., Vervoort, J., and Rietjens, I. M. C. M., TEAC antioxidant activity of 4-hydroxybenzoates. Free Radic. Biol. Med., 27, 14271436 (1999).

5) Kimura, T., Yamamoto, S., Ogawa, I., Miura, H., and Hasegawa, M., Antioxidant ability of chicoric acid and its analogous compounds. Nippon Kagaku Kaishi, 739 750 (1999).

6) Kawabata, J., Okamoto, Y., Kodama, A., Makimoto, T., and Kasai, T., Oxidative dimers produced from protocatechuic and gallic esters in the DPPH radical scavenging reaction. J. Agric. Food Chem., 50, 5468-5471 (2002).

7) Saito, S., Okamoto, Y., Kawabata, J., and Kasai, T., Quinone hemiacetal formation from protocatechuic acid during DPPH radical scavenging reaction. Biosci. Biotechnol. Biochem., 67, 1578-1579 (2003).

8) Sayre, L. M., and Nadkarni, D. V., Direct conversion of phenols to $o$-quinones by copper (I) dioxygen. Questions regarding the monophenolase activity of tyrosinase mimics. J. Am. Chem. Soc., 116, 3157-3158 (1994).

9) Saito, S., Okamoto, Y., and Kawabata, J., Effects of alcoholic solvents on antiradical abilities of protocatechuic acid and its alkyl esters. Biosci. Biotechnol. Biochem., 68, 1221-1227 (2004).

10) Saito, S., and Kawabata, J., Synergistic effects of thiols and amines on antiradical efficiency of protocatechuic acid. J. Agric. Food Chem., 52, 8163-8168 (2004).
11) Saito, S., Gao, H., and Kawabata, J., DPPH (=2,2-diphenyl-1-picrylhydrazyl=2,2-diphenyl-1-(2,4,6-trinitrophenyl)hydrazyl) radical-scavenging reaction of protocatechuic acid esters ( $=3,4$-dihydroxybenzoates) in alcohols: formation of bis-alcohol adduct. Helv. Chim. Acta, 89, 821-831 (2006).

12) Feldman, K. S., Quideau, S., and Appel, H. M., Galloylderived orthoquinones as reactive partners in nucleophilic additions and Diels-Alder dimerizations: a novel route to the dehydrodigalloyl linker unit of agrimoniintype ellagitannins. J. Org. Chem., 61, 6656-6665 (1996).

13) Quideau, S., and Feldman, K. S., Ellagitannin chemistry. The first synthesis of dehydrohexahydroxydiphenoate esters from oxidative coupling of unetherified methyl gallate. J. Org. Chem., 62, 8809-8813 (1997).

14) Bhatia, I. S., Nagpal, M. L., Singh, P., Kumar, S., Singh, N., Mahindra, A., and Parkash, O., Chemical nature of the pigment of the seed coat of guar (cluster bean, Cyamopsis tetragonolobus L. Taub). J. Agric. Food Chem., 27, 1274-1276 (1979).

15) Gorinstein, S., Weisz, M., Zemser, M., Tilis, K., Stiller, A., Flam, I., and Gat, Y., Spectroscopic analysis of polyphenols in white wines. J. Ferment. Bioeng., 75, 115-120 (1993).

16) Li, G., Min, B.-S., Zheng, C., Lee, J., Oh, S.-R., Ahn, K.-S., and Lee, H.-K., Neuroprotective and free radical scavenging activities of phenolic compounds from Hovenia dulcis. Arch. Pharm. Res., 28, 804-809 (2005).

17) Blois, M. S., Antioxidant determinations by the use of a stable free radical. Nature, 181, 1199-1200 (1958).

18) Brand-Williams, W., Cuvelier, M. E., and Berset, C., Use of a free radical method to evaluate antioxidant activity. Lebensm. Wiss. Technol., 28, 25-30 (1995).

19) Quideau, S., Feldman, K. S., and Appel, H. M., Chemistry of galloyl-derived o-quinones: reactivity toward nucleophiles. J. Org. Chem., 60, 4982-4983 (1995).

20) Saito, S., and Kawabata, J., DPPH (=2,2-diphenyl-1picrylhydrazyl) radical-scavenging reaction of protocatechuic acid (=3,4-dihydroxybenzoic acid): difference in reactivity between acids and their esters. Helv. Chim. Acta, 89, 1395-1407 (2006). 